\title{
Crossed fused renal ectopia with renal calculus and gross hydronephrosis- a case report
}

\begin{abstract}
Crossed renal ectopia is a rare congenital malformations where a kidney is located on the side opposite to side of its urethral insertion into the urinary bladder and is generally fused with the normally located ipsilateral kidney.1 Resulting from aberrant migration and crossing of the midline of the metanephric blastema and ureteral bud, CFRE is thought to develop during $4^{\text {th }}$ to $8^{\text {th }}$ weeks of gestation. Mostly remaining asymptomatic and detected as an incidental finding during imaging studies, six welldefined anatomical variations of CFRE have been reported.2 We report here one such case of crossed fused renal ectopia detected by USG, which was later on confirmed by computed tomography
\end{abstract}

Keywords: CFRE, MDCT
Volume 4 Issue 6 - 2017

Aeshita Singh
Apollo hospital, India

Correspondence: Aeshita Singh, Apollo hospital, Old high court road, Bilspur, Chhattisgarh, India, Tel 8982342723 , Email aeshita17@gmail.com

Received: August 27, 2017 | Published:November 29, 2017

\section{Introduction}

Failure of kidney to ascend during embryologic development results in a pelvic kidney. Prevalence rate of crossed fused renal ectopia 1 in 724 paediatric autopsies. These kidneys are often small and abnormally rotated. The ureters are often short; poor drainage and dilatation of collecting system predispose to secondary infection and calculi formation. The blood supply is often complex; multiple arteries may be derived from regional arteries like the internal iliac artery or the common iliac artery. ${ }^{3}$

Crossed fused ectopia of the kidney is a rare malformation occurring in $0.05-0.1 \%$ of the population. ${ }^{4}$ It is more common in males with a 2:1 male to female ratio. Ectopic kidney will be fused to the other kidney In $85-90 \%$ of cases. The upper pole of the ectopic kidney is usually fused with the lower pole of the other kidney. On ultrasound, both kidneys are on the same side and are typically fused with a characteristic anterior or posterior "notch" between the two fused kidneys (Figure 2) with a difference in orientation of the 2 collecting systems in the fused kidneys. ${ }^{5,6}$

\section{Case presentation}

69year-old female was referred to outpatient clinic of our hospital with complaints of chest pain, restlessness and giddiness since two days. Her initial abdominal ultrasonography had reported renal ectopia with both kidneys located in the right side, left kidney was absent in left renal fossa. Routine laboratory studies did not document any abnormalities (i.e., blood urea nitrogen: $28 \mathrm{mg} / \mathrm{dL}$, creatinine: $1.4 \mathrm{mg}$ / $\mathrm{dL}$, normal serum electrolyte concentrations, normal urinalysis). A repeated renal ultrasound documented left ectopic kidney, right located superiorly and left located inferiorly (Figure 1). Renal dimensions of the superiorly and the inferiorly situated kidneys were $9 \times 4.3 \mathrm{cms}$ and $11.6 \times 6.2 \mathrm{cms}$, respectively. The superiorly located kidney also displayed irregular contours and a thinner parenchyma, with multiple renal calculi and dilatation of collecting systems was observed (Figure 3-5). Later on CT KUB was done in which lobulated outline of right kidney with multiple calculi and left kidney was found absent in left renal fossa with ectopically malrotated lower uretric regions (Figure 2 \& Figure 5).

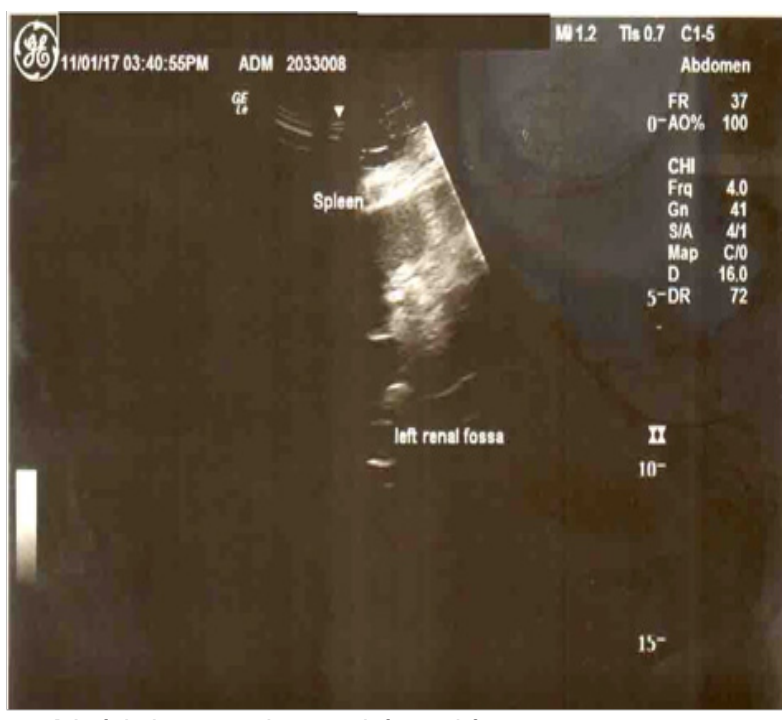

Figure I Left kidney was absent in left renal fossa.

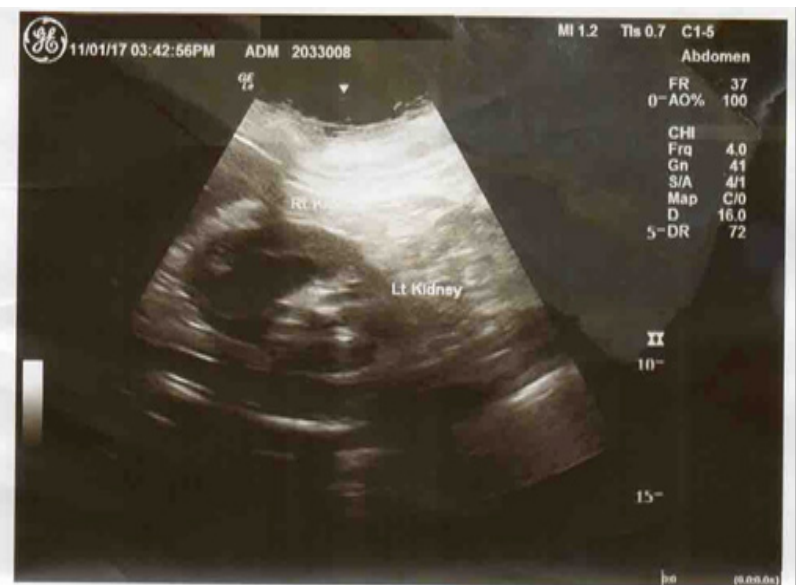

Figure 2 Both right and left kidneys were fused, right kidney was located superiorly with lobulated margins and hydronephrosis whereas left kidney was located inferiorly, multiple calculi was demonstrated in both kidneys. 


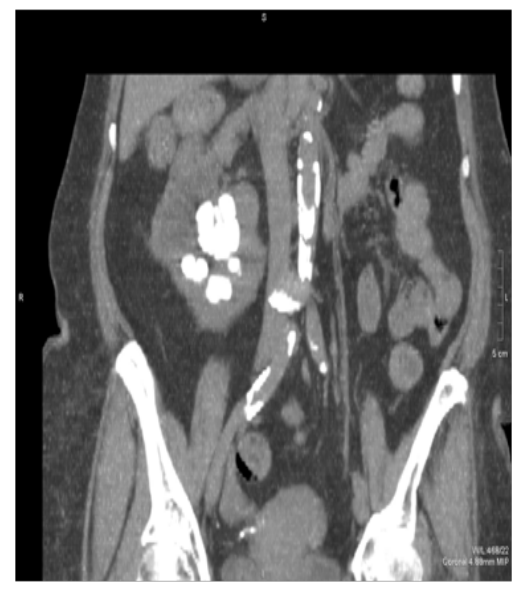

Figure 3 Coronal CT images showing fused right and left kidneys, right kidney was located superiorly with lobulated margins and hydronephrosis where as left kidney was located inferiorly, multiple calculi was demonstrated in both kidneys.

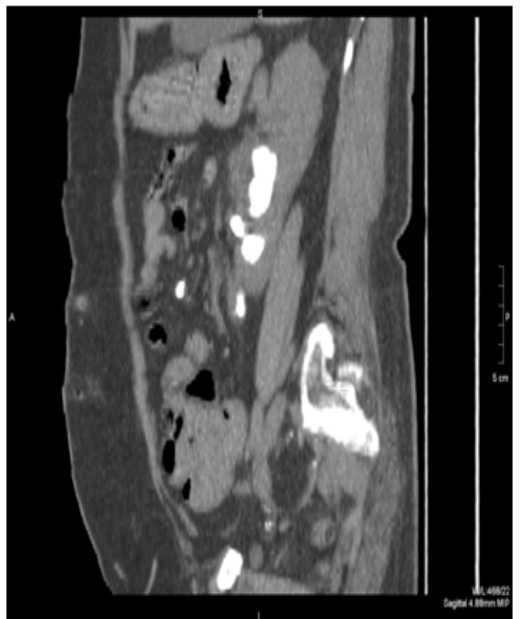

Figure 4 Coronal CT images showing fused right and left kidneys, right kidney was located superiorly with lobulated margins and hydronephrosis where as left kidney was located inferiorly, multiple calculi was demonstrated in both kidneys.

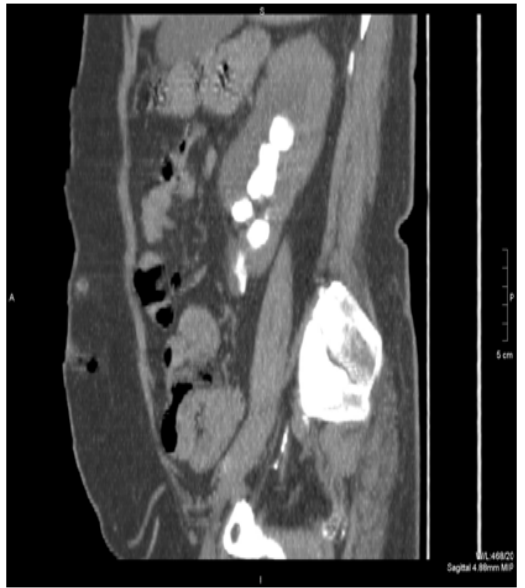

Figure 5 Coronal CT images showing fused right and left kidneys, right kidney was located superiorly with lobulated margins and hydronephrosis where as left kidney was located inferiorly, multiple calculi was demonstrated in both kidneys.

\section{Discussion}

Crossed fused renal ectopia is a congenital malformation that is present at birth. In the absence of associated complications and symptoms, the condition may be incidentally discovered on images obtained for reasons other than the evaluation of crossed fused kidneys. Many cases of crossed fused renal ectopia remain undiagnosed. At times deformed kidney that results from a neoplasm or previous trauma in addition to congenital absence of the contralateral kidney may be mistaken for crossed fused renal ectopia. Non-functioning of one of fused renal units (e.g, as a result of obstruction) may cause crossed fused ectopia to be missed. Upto $50 \%$ of ectopic kidneys are at least partially blocked. Overtime, obstruction can lead to serious complications, including urinary tract infections, kidney stones and kidney failure. Both stag horn and spiked calculi were seen in this case. Urinary tract stones composed of calcium oxalate dihydrate can assume a spiked configuration, resembling a child's toy jack. ${ }^{7}$ McDonald and McClellan classified crossed ectopic kidney into four types: crossed renal ectopia with fusion (85\%), crossed renal ectopia without fusion $(10 \%)$, solitary crossed renal ectopia, and bilaterally crossed renal ectopia. ${ }^{8}$ Six variations of crossed fusion have been described. In decreasing order of frequency, they are: type 1, inferior crossed fused ectopia; type 2, sigmoid or S-shaped kidney; type 3, unilateral lump kidney; type 4, unilateral disc kidney; type 5, L-shaped kidney; type 6 , superior crossed fused ectopia. ${ }^{9}$

Review of literature showed only 6 cases of stone disease treated in crossed renal ectopic kidneys. The anomalous position of kidneys and abnormal disposition of arterial supply pose a different surgical challenge, requiring a careful definition of renal outlines by contrast enhanced CT scans, mapping of vasculature by arteriography and collecting system with drainage pattern by cystoscopy and bilateral ureteropyelography. ${ }^{10}$

\section{Conclusion}

CFRE is the second most common FAs of the kidneys after HK. CFRE results from one kidney crossing over to

the opposite side and subsequent fusion of the parenchyma of the two kidneys. Usually, CFRE is diagnosed as an incidentally, when patient is examined for other reasons, unless it is complicated by infection or obstruction. MDCT examination can provide good delineation of vascular and urinary tract anomalies. In CFRE, the arterial supply and venous drainage are grossly abnormal, in correlations with the incomplete ascent of the kidneys and with the primitive arrangement of the vascular and urinary tract structures. The interventional radiological and surgical procedure must be planned according to the anatomical variations of the arterial, venous and urinary tract structures of each case of CFRE. ${ }^{11}$

\section{Acknowledgements}

None.

\section{Conflict of interest}

Author declares that there is no conflict of interest.

\section{References}

1. Sharma V, Ramesh Babu CS, Gupta OP. Crossed fused renal ectopia multidetector computed tomography study. International Journal of Anatomy and Research. 2014;2(2):305-309. 
2. Leyla Akdogan, Ali Kemal Oguz, Tarkan Ergun, et al. The Rarest of the Rare: Crossed Fused Renal Ectopia of the Superior Ectopia Type. Case Reports in Nephrology. 2015;2015:4

3. Friedland GW, Devries PA, Nino-Murcia M. Clinical urography: an atlas and text book of urologic imaging. Congenital anomalies of the urinary tract. 1990;1990:559-787.

4. Modi P, Rizvi SJ, Gupta R, et al. Retroperitoneoscopic nephrectomy for crossed-fused ectopic kidney. Indian J Urol. 2009;25(3):401-403.

5. Yuksel A, Batukan C. Sonographic findings of fetuses with an empty renal fossa and normal amniotic fluid volume. Fetal Diagn Ther. 2004;19(6):525-532.

6. Goodman JD, Norton KI, Carr L, et al. Crossed fused renal ectopia: sonographic diagnosis. Urol Radiol. 1986;8(1):13-16.
7. Dyer RB, Chen MY, Zagoria RJ. Uroradiologic signs. Classic signs in uroradiology. Radiographics. 2004;24:247-280.

8. McDonald JH, McClellan DS. Crossed renal ectopia. Am J Surg. 1957;93:995-999.

9. Bauer SB. Anomalies of the upper urinary tract. In: Walsh PC, editors. Campbell's urology. 8th ed. Philadelphia: WB Saunders; 2002. p. 1898-1906.

10. Nabi G, Kasana I, Khan M. Management of nephrolithiasis in crossed renal ectopia. J Postgrad Med. 2001;47(1):66-67.

11. Pupca G, Miclăuş GD, Bucuraş V, et al. Left crossed fused renal ectopia L-shaped kidney type, with double nutcracker syndrome (anterior and posterior). Rom J Morphol Embryol. 2014;55(3 Suppl):1237-1241. 